\title{
Participatory Learning of Medical Students through Development of Innovative Training Modules for Community Health Workers
}

Rahul Ramesh Bogam, ' Vivek Saoji, ${ }^{2}$ Ranjana Sahasrabudhe, ${ }^{2}$ Apoorva Saoji. ${ }^{2}$

\begin{abstract}
Background: Community Medicine is a subject which receives relatively less attention in medical curriculum. Active participation of students in preparation of training modules for Community Health Workers (CHWs) could facilitate their learning in topics related to Community Medicine. This study aimed to involve medical students in the preparation of training modules for CHWs and assess the effects of their participation on their knowledge about targeted diseases. Methods: An interventional study where all 144 undergraduate fifth-semester students from Bharati Vidyapeeth University Medical College, Pune, India posted at Community Medicine Department participated in the preparation of training modules for CHWs on HIV/AIDS, tuberculosis, malaria and diabetes and completed pre- and post-intervention questionnaire. Each completed questionnaire was assigned a score based on a marking system. The data was analysed using paired $t$ test. Results: Statistically significant improvement in knowledge was found (pre-test mean score: 5.79, post-test mean score: 14.15, $t=26.93$, p<0.001). All faculty opined that community visits followed by health education module preparation activity were innovative components in this study. All 119 participants agreed that their knowledge about targeted diseases improved due to module preparation activity. Conclusion: Active participation of students can facilitate their learning behavior. Students became aware of the principles of 'Group Dynamics' as a result of active involvement in training module preparation.
\end{abstract}

Keywords: Students, Medical; Education, Medical; Community Medicine; Community Health Workers; Health Education (Source: MeSH-NLM).

About the Author: Apoorva Saoji is an undergraduate medical student at B harati Vidyapeeth University Medical College, Pune, Maharashtra, India.

\section{Introduction}

Community Medicine is one of the essential subjects in medical curriculum. However this subject is identified as less prioritized in medical curriculum especially by first and second year medical students in Indian medical schools.' Some of the perceptions of undergraduate students leading to their poor involvement in this subject are 'lack of relevance', 'overemphasis on cognitive learning,' and 'vast and uninteresting subject'.' Active participation of students facilitates learning. ${ }^{2}$ Hence it was decided to involve undergraduate students actively and promote learning through their active participation in community oriented activities.

Community Health Workers (CHWs) like Accredited Social Health Activists (ASHAs) and Anganwadi Workers (AWWs) are local members of the community and serve as a crucial link between health system and the community. ${ }^{3}$ They create awareness, encourage, and help people to access health and related services such as immunization, antenatal check ups, postnatal follow ups, supplementary nutrition, sanitation, health education and other services being provided by the government which area available at the Anganwadi Subcentre / Primary Health Centre. ${ }^{4}$ Many reports acknowledge the contribution by $\mathrm{CHWs}$ in the decline of vector borne diseases like malaria and other public health problems like tuberculosis and water borne diseases. ${ }^{4}$
CHWs are usually from among the community and have limited knowledge of the various health and related issues. The role of health education training modules to sensitize them about various diseases is crucial for making them good trainers to reduce the magnitude of these diseases in the community. Few studies have reported serious difficulties faced by CHWs in understanding the health education manuals; therefore, there is a strong need to make simple, innovative and easy to understand health education training modules for them. ${ }^{3}$

Diseases like tuberculosis, HIV/AIDS, malaria and diabetes mellitus are prevalent public health problems particularly in a developing country like India. These diseases have been identified as significant causes of morbidity and mortality, particularly in areas with low literacy levels. ${ }^{5}$ These diseases are also essential components in the undergraduate (UG) medical curriculum. The UG students need to be sensitized about preventive as well as curative aspects of these diseases from the beginning of the course. Public health experts also emphasize the need for active fieldwork collaboration between medical students and CHWs to control diseases of public health concern. We therefore decided to involve UG medical students in preparing training modules on these health problems. The present study was thus conducted to develop low cost, simple, innovative health education modules for CHWs on targeted diseases. With the aid

Department of Family and Community Medicine, College of Medicine, King Faisal University, Saudi Arabia. Bharati Vidyapeeth University Medical College, Pune, Maharashtra, India. 
of active participation of undergraduate medical students, the aim of the competency-based modules could be used to train CHWs at a wide range of education levels. Further these modules can be a potential source to disseminate accurate, complete and easily understandable information about these diseases to CHWs. To the best of our knowledge, so far no study has been undertaken to evaluate the effect of involvement of medical students in the preparation of health education modules for CHWs.

The aim of this study were to: (1) develop simple, low cost, innovative health education modules for Community Health Workers through active student's participation; (2) assess the effect of active participation on undergraduate medical students in terms of their knowledge of the specific topics as well as interest in the subject of Community Medicine; and (3) evaluate this educational intervention through feedback from all participants including students and faculty.

\section{Methods \\ Participants}

An interventional study was conducted at Bharati vidyapeeth University Medical College, Pune, Maharashtra State, India. A total of 144 undergraduate MBBS medical students in their fifth semester (second year MBBS) who were posted in the Community Medicine Department participated in the study. They were divided into four batches (A, B, C and D) of 36 students each. Informed consent was taken from all participants. Ethical committee approval was obtained from the Institutional Ethics Committee. (Ref.No - BVDU/MC/83).

\section{Questionaire}

After literature review, structured questionnaires were developed, each on the four selected common public health problems, namely, malaria, tuberculosis, HIV/AIDS and diabetes mellitus. The questionnaire was designed to assess knowledge of the UG students about these topics and were validated with the help of expert faculty. Each questionnaire comprised of 20 multiple-choice, single-best-answer type questions.

\section{Phase I Intervention}

Orientation session. An orientation session was organized for study participants to sensitize them about the project including information about targeted diseases (malaria, tuberculosis, HIV/AIDS and diabetes mellitus), need for simple innovative modules for CHWs and tentative structure of modules like aetiology of disease, diagnosis, treatment and prevention were discussed with the students. Existing health education modules on these diseases were given to them to assess the need for new, innovative training modules.

Since the participants were expected to work in groups, a brief session on 'Croup Dynamics' was conducted in order to facilitate smooth working of groups. Each group identified one group leader by consensus for smooth coordination.

Each batch of participants was further divided into four sub groups of nine students each. On the same day, the participants were asked to answer a structured pre-validated questionnaire to judge their basic knowledge about diseases to be covered in the project. These scores were considered as pre-test scores.
Community exposure. Before commencement of the study, all participants were exposed to field practice areas of the institute as a part of 'Community Visit' to do situational analysis of targeted diseases.

Use of innovative teaching methodologies. As per need assessment done by participants for the training modules, it was decided to incorporate 'Role Play', 'Puppet Show', 'Games' and 'Slogans' for the easy understanding of the diseases by CHWs. All participants were given two days training on 'Script Writing' and 'How to conduct role play' by trained faculty.

\section{Phase II Intervention}

Preparation of training modules. The students under faculty supervision developed the training modules in the local (Marathi) language on four themes i.e. malaria, tuberculosis, HIV/AIDS and diabetes mellitus. Each group was assigned a specific task of preparing one module on each theme during the one month posting period. Faculty members provided guidance during the student's preparation by reviewing their work each week. During the final week of the posting, students presented their work and modules were modified according to the suggestions made by faculty members. A total of 4 modules were developed by the end of the study.

Role play and slogan competition. All four groups participated in 'Role Play and Slogan Competition' wherein they actually performed in the theme based role play competition and also submitted five slogans on the selected themes.

\section{Post-Intervention}

At the end of the posting, the same pre-intervention questionnaire was administered to all participants as a post test and responses were collected. Scores obtained in this test were considered as 'Post Test' scores. The pre and post test scores were entered in a Microsoft Office Excel Sheet and analysed by using the 'Paired t test'.

\section{Results}

All 144 participants belonging to semester five, with an age range of 20 to 24 years participated in the study. Out of these, 25 participants did not appear for the post-test and were excluded from the study. Hence data analysis was completed for 119 participants who completed the study.

Mean pre and post intervention scores show that active participation in preparing training modules significantly enhanced the knowledge of participants about the diseases covered $(t=26.93$, $p<0.001$ ).

All faculty opined that community visits followed by health education module preparation activity and need based assessment were innovative components in this entire study. The need to expand this study to address other public health problems in the community was reiterated by all of the faculty. Three faculty members (50\%) reported local (Marathi) language difficulty perceived by participants and felt the need to make them well versed with the local language. However, one faculty member $(16.66 \%)$ said that it may be beneficial to take lessons from a professional language translator as students may get difficulties in language translation i.e. English to Marathi while preparing modules. 
All faculty felt that involving students directly in this type of community oriented activity and giving them responsibility to prepare training modules was an innovative idea. All 119 participants agreed that their knowledge about the four targeted diseases was improved due to the module preparation and other interactive activities.

From the present study, four low cost, innovative and easily understandable training modules were developed in local language on malaria, tuberculosis, HIV/AIDS and diabetes mellitus. Emerged modules included a wide array of innovative teaching methods like 'Catchy Slogans' and 'Interesting scripts of Role Play' which were considered as 'Best Part of study' by 114 (95.80\%) participants (Table 1). All faculty members also mentioned in the feedback that these new modules should be used not only to train CHWs but also general people in community about targeted four diseases.

\section{Discussion}

Contemporary medical education emphasizes the 'SPICES' model (Student centred learning, Problem based learning, Integrated curricula, Community based learning, Electives with a core, and Systematic curricula) where there is need to shift from 'Hospital Based Education' to 'Community Based Education' and 'Teacher Centred' to 'Student Centred'. Harden et al. (1984) reiterated that the hospital based learning has fostered an 'Ivory Tower' approach to medicine in which, during their training, students have little contact, if any, with the communities which they are being trained to serve. ${ }^{6}$

Involving students in community oriented participatory activities is a useful method to engage them in community based practices as well as to assess health needs of the community. Rao et al. (2007) also reported successful student integration in community based participatory research.?

The aim of the present study was to ensure active participation of students in community oriented projects at an early stage of training in order to facilitate their learning and to generate an interest in the subject of 'Community Medicine'. Towards this aim, high engagement, self-learning activities were used in the form of games, skits, slogan competitions, puppet shows, and quizzes along with module preparation helped to facilitate the learning of students.

Rezende-Filhoet al. (2014) reported active and meaningful learning among students during preparation of various physiological-physical models. ${ }^{8}$ Sward et al. (2008) used web based games to teach pediatric content to medical students and it was found to be effective. ${ }^{9}$ Sprengel (1994) also effectively used games for teaching students. ${ }^{10}$

The present study reiterates that active involvement of stu-

Table 1. Feedback of Study Participants about the Entire Study $(n=119)$

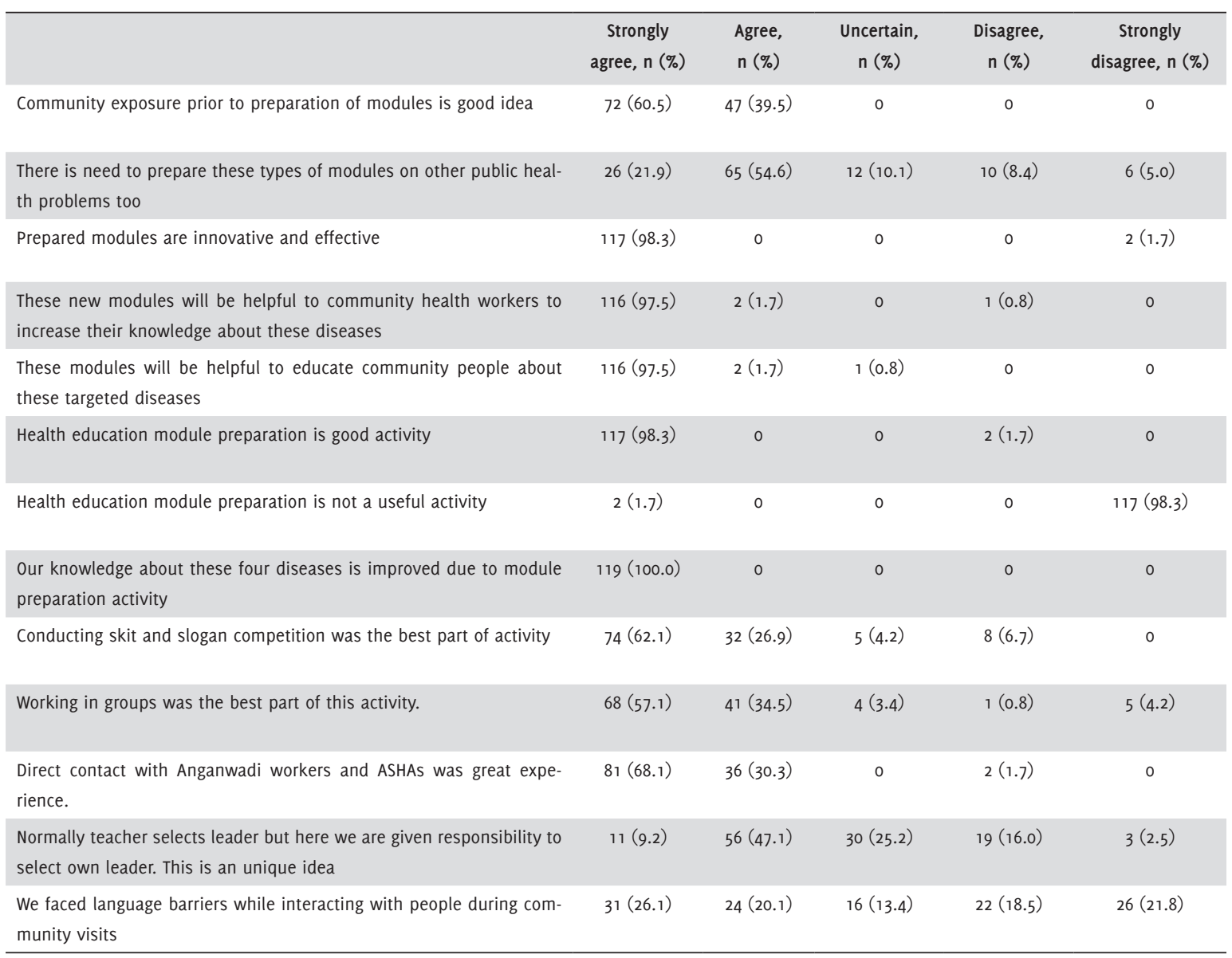


dents in the module preparation process along with their engagement in other self-learning activities enhanced their knowledge about four important public health problems which form a vital part of their curriculum, namely tuberculosis, HIV/AIDS, malaria and diabetes mellitus. Wankat (2002) reported multiple studies which suggest that student involvement is one of the most important predictors of success in college." Hake assessed pre and post test data of 6000 students in introductory science courses and observed statistically significant improvement in student performance due of interactive engagement methods. ${ }^{12}$

The present study helped students to develop a positive attitude towards 'Community Medicine' as well as community oriented participatory research, as indicated by their written feedback. Overall feedback reflects that students enjoyed the process of module preparation and other interactive activities like 'Role Plays and Slogans'.

This underlines the need to motivate students for participation in community oriented activities and/or research at an early stage of training. When community oriented research projects and students are suitably matched, it not only enhances the potential of community benefits from the project, but also encourages the development of future community physicians and leaders.

The present study showed significant improvement in the student's knowledge about targeted diseases. It indicates that active participation of students can facilitate their learning behavior. Students also became aware about the principles of 'Croup Dynamics' and the proactive self-study helped to develop an interest in subject.

The modules prepared as an outcome of this project will now be used to train Community Health Workers in rural and urban areas about the four targeted public health problems, namely tuberculosis, malaria, HIV/AIDS and diabetes mellitus. These trained CHWs would subsequently use the same modules to educate the general community about these four important public health problems. It is further planned to evaluate the impact of the modules on the knowledge and skills of CHWs and its impact on the health of the community. There are also future plans to develop additional modules for self-study by involving and engaging students, thereby fostering active learning.

\section{References}

1. Kumar P. Teaching community medicine to undergraduates, problems and solutions: a loud thinking. Natl J Community Med. 2013 Jan-Mar;4(1):1-3.

2. Tejinder S, Piyush G, Daljit S. Principles of medical education.4th ed. New Delhi: Jaypee Brothers Medical Publishers; 2013.

3. Prasad BM, Muraleedharan VR. Community health workers: a review of concepts, practice and policy concerns. International Consortium for Research on Equitable Health Systems; 2007.

4. Global Health Workforce Alliance, World Health Organization. Clobal experience of community health workers for delivery of health related millennium development goals. a systematic review, country case studies, and recommendations for integration into national health systems. Ceneva: World Health Organization; 2010.

5. Park K. Textbook of preventive and social medicine. 23 rd ed. Jabalpur (India): Banarsidas Bhanot Publishers; 2015.

6. Harden RM, Sowden S, Dunn WR. Educational strategies in curriculum development: the SPICES Model. Med Educ. 1984 July;18(4):284-97.

7. Rao P, Arcury TA, Quandt SA. Student participation in community-based participatory research to improve migrant and seasonal farmworker environmental health: issues for success. J Environ Educ. 2004 Jan;35(2):3-15.

8. Rezende-Filho FM, da Fonseca LJ, Nunes-Souza V, Guedes Gda S, Rabelo LA. A student-centered approach for developing active learning: the construction of physical models as a teaching tool in medical physiology. BMC Med Educ. 2014 Sep 15;14:189.

9. Sward KA, Richardson S, Kendrick J, Maloney C. Use of a Web-based game to teach paediatric content to medical students. Ambul Pediatr. 2008 NovDec;8(6):354-9.

10. Sprengel AD. Learning can be fun with gaming. J Nurs Educ. 1994 Apr;33(4):151-2.

11. Wankat PC. The effective efficient professor: teaching, scholarship and service. Boston: Allyn and Bacon; 2002.

12. Hake R. Interactive-engagement vs. traditional methods: a six-thousand-student survey of mechanics test data for introductory physics courses. Am J Phys. 1998 Jan;66(1):64-74.

\footnotetext{
Acknowledgments

We are thankful to all faculty members and supporting staff of the Department of Community Medicine, Bharati Vidyapeeth University Medical College, Pune, India for their valuable help in implementation of this study.

Conflict of Interest Statement at Funding

This study was funded by Bharati Vidyapeeth University Medical College, Pune, India.

\section{Author Contributions}

Conceptualization, Writing, Critical revision of the manuscript: RRB, VS, RS. Data collection: AS. Approval of the final version: RRB, VS, RS, AS. Funding acquisition: VS.

Cite as:

Bogam RR, Saoji V, Sahasrabudhe R, Saoji A. Participatory learning of medical students through development of innovative training modules for community health workers. Int J Med Students. 2016 Sep-Dec;4(3):100-3.
} 